\title{
Stability of a Light Sail Riding on a Laser Beam
}

\section{Citation}

Manchester, Zachary, and Abraham Loeb. 2017. "Stability of a Light Sail Riding on a Laser Beam." The Astrophysical Journal 837 (2) (March 7): L20. doi:10.3847/2041-8213/aa619b.

\section{Published Version}

doi:10.3847/2041-8213/aa619b

\section{Permanent link}

http://nrs.harvard.edu/urn-3:HUL.InstRepos:32751018

\section{Terms of Use}

This article was downloaded from Harvard University's DASH repository, and is made available under the terms and conditions applicable to Open Access Policy Articles, as set forth at http:// nrs.harvard.edu/urn-3:HUL.InstRepos:dash.current.terms-of-use\#OAP

\section{Share Your Story}

The Harvard community has made this article openly available.

Please share how this access benefits you. Submit a story.

\section{Accessibility}


Draft version December 21, 2016

Preprint typeset using $\mathrm{LAT}_{\mathrm{E}} \mathrm{X}$ style emulateapj v. 01/23/15

\title{
STABILITY OF A LIGHT SAIL RIDING ON A LASER BEAM
}

\author{
ZACHARY MANCHESTER \\ John A. Paulson School of Engineering and Applied Science, Harvard University, 60 Oxford St., Cambridge, MA 02138
}

ABraham Loeb

Astronomy Department, Harvard University, 60 Garden St., Cambridge, MA 02138

Draft version December 21, 2016

\begin{abstract}
The stability of a light sail riding on a laser beam is analyzed both analytically and numerically. Conical sails on Gaussian beams, which have been studied in the past, are shown to be unstable without active control or additional mechanical modifications. A new architecture for a passively stable sail and beam configuration is proposed. The novel spherical shell design for the sail is capable of "beam riding" without the need for active feedback control. Full three-dimensional ray-tracing simulations are performed to verify our analytical results.
\end{abstract}

\section{INTRODUCTION}

The light sail concept-harnessing photon pressure to propel a spacecraft-has a long history dating back to some of the earliest pioneers of astronautics. Tsiolkovsky \& Zander first described "tremendous mirrors of very thin sheets... using the pressure of sunlight to attain cosmic velocities" in 1925 (Zander 1964). Since then, most research has focused on solar sails-light sails that harness solar photons. Following the invention of lasers, in the 1960s Forward (Forward 1984), Marx (Marx 1966), and Redding (Redding 1967) independently proposed the use of high-power lasers as a means of propelling a sail to a significant fraction of the speed of light. This was followed by subsequent studies over the past five decades (Moeckel 1972, Weiss et al. 1979; Lubin shed). Most recently, the Breakthrough Starshot Initiative ${ }^{1}$ was funded to propel a gram-scale spacecraft attached to a sail to a fraction of the speed of light using a high-power laser, with the goal of reaching the nearest stars within several decades.

There are many difficult engineering challenges associated with laser-propelled light sails that remain to be solved. A particularly important problem is ensuring that the sail remains centered on the laser beam despite disturbances, misalignment, and manufacturing imperfections. Ideally, a sail should possess beam-riding stability without the need for active feedback control, as the addition of sensor and actuator hardware would add significant complexity and mass to the spacecraft.

While a substantial literature exists on the stability and control of solar sails (Wie 2004a b; Smith et al. 2005; Mimasu et al. 2011; Polites et al. 2008), laser-propelled sails have received far less attention. 'The most closely related previous work has focused on conical microwavepropelled sails, which were studied both in numerical simulations (Chahine et al. 2003: Schamiloglu et al. 2001) and laboratory experiments (Benford et al. 2002, 2003). However, a rigorous theoretical analysis of the stability of such sails was not performed.

zmanchester@seas.harvard.edu

aloeb@cfa.harvard.edu

1 http://breakthroughinitiatives.org/Initiative/3
This paper analyzes the beam-riding stability of laserpropelled light sails and proposes a new passively stable laser and sail configuration. Section 2 provides an introduction to the beam-riding problem and reviews some basic results from linear stability theory. Next, Section 3 derives a linearized dynamical model of a conical sail riding a Gaussian laser beam. Section 4 then uses the model to show that such sail configurations are unstable without active feedback control or mechanical modifications. In Section 5 we propose a novel passively stable spherical sail architecture. Section 6 presents the results of numerical ray-tracing simulations that demonstrate the stability of the proposed design. Finally, Section 7 summarizes our results and offers some commentary on future research directions.

\section{BACKGROUND}

\subsection{The Beam-Riding Problem}

The basic features of the beam-riding problem are depicted in Figure 1. A laser beam of width $W$ is incident on a sail of radius $R$, where $W$ and $R$ are assumed to be of the same order of magnitude. The challenge isthrough shaping the sail, choosing its composition, and possibly using active feedback control-to keep the sail centered on the beam as it is accelerated.

The total force applied by a beam incident on a perfectly reflective sail of area $S$ is given by,

$$
\boldsymbol{F}=\int_{S} 2 \frac{P(\boldsymbol{x}) \hat{\boldsymbol{b}} \cdot \hat{\boldsymbol{n}}(\boldsymbol{x})}{c} \hat{\boldsymbol{n}}(\boldsymbol{x}) d S,
$$

where the domain of integration is the surface of the sail, $\hat{\boldsymbol{n}}(\boldsymbol{x})$ is the unit vector normal to the sail surface at the point $\boldsymbol{x}, P(\boldsymbol{x})$ is the beam power flux at the point $\boldsymbol{x}, \hat{\boldsymbol{b}}$ is a unit vector parallel to the beam axis, and $c$ is the speed of light. Similarly, the total torque applied by the beam to the sail is given by,

$$
\boldsymbol{\tau}=\int_{S} 2 \frac{P(\boldsymbol{x}) \hat{\boldsymbol{b}} \cdot \hat{\boldsymbol{n}}(\boldsymbol{x})}{c}(\boldsymbol{r}(\boldsymbol{x}) \times \hat{\boldsymbol{n}}(\boldsymbol{x})) d S,
$$

where $\boldsymbol{r}(\boldsymbol{x})$ is the vector from the sail's center of mass to point $\boldsymbol{x}$. If the sail is assumed to be rigid, its motion can 


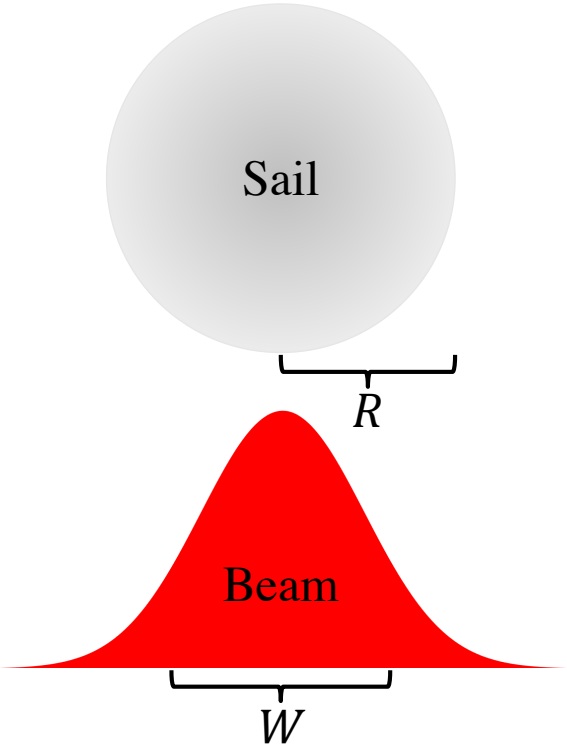

Figure 1. Schematic illustration of the beam-riding problem.

be described by Newton's second law,

$$
m \ddot{\boldsymbol{x}}=\boldsymbol{F},
$$

and Euler's equation,

$$
I \dot{\omega}+\omega \times I \omega=\tau,
$$

where $m$ is the mass, $\boldsymbol{\omega}$ is the angular velocity vector, and $I$ is the inertia tensor of the sail.

Analyzing the motion of a sail based on equations (1) (4) is difficult; the integrals in equations (1) and (2) cannot, in general, be computed in closed form, and the differential equation describing angular motion (4) is nonlinear. In the subsequent sections, we will attempt to side-step some of these analytical difficulties to obtain general stability characterizations of various beam-riding configurations.

\subsection{Linear Stability Analysis}

We start by briefly reviewing the mathematical formalism of stability theory. For thorough treatments, the reader is referred to references (Kailath 1980) and (Hirsch et al. 2003).

A nonlinear dynamical system can be generically written as a first-order vector differential equation,

$$
\dot{\boldsymbol{x}}=\boldsymbol{f}(\boldsymbol{x}),
$$

where $\boldsymbol{x} \in \mathbb{R}^{n}$ is the state vector of the system. Differential equations involving higher-order derivatives can always be put into this form by introducing additional variables (Hirsch et al. 2003). An equilibrium point of the system is a point $\boldsymbol{x}^{*}$ such that,

$$
\boldsymbol{f}\left(\boldsymbol{x}^{*}\right)=0 \text {. }
$$

Without loss of generality, we will assume that $\boldsymbol{x}^{*}$ coincides with the origin.

A linear dynamical system is described by a square matrix $A \in \mathbb{R}^{n \times n}$ such that,

$$
\dot{\boldsymbol{x}}=A \boldsymbol{x} .
$$

Nonlinear systems can be approximated in the neighborhood of the origin by taking $A$ to be,

$$
A_{i j}=\frac{\partial f_{i}}{\partial x_{j}} .
$$

Solutions of (7) are given by,

$$
\boldsymbol{x}(t)=e^{A t} \boldsymbol{x}_{0},
$$

where $\boldsymbol{x}_{0}$ is a vector of the initial conditions and $e^{A t}$ is a matrix exponential, which is defined in terms of the power series of the exponential function (Kailath 1980).

If $A$ can be decomposed such that $A=V D V^{T}$, where $V$ is a matrix whose columns are the eigenvectors $\boldsymbol{v}_{i} \in \mathbb{R}^{n}$ of $A$, and $D$ is a diagonal matrix whose entries are the eigenvalues $\lambda_{i} \in \mathbb{C}$ of $A$, then equation (9) takes the particularly simple form,

$$
\boldsymbol{x}(t)=V e^{D t} V^{T} \boldsymbol{x}_{0}=\sum_{i=1}^{n} \boldsymbol{v}_{i} e^{t \lambda_{i}} \boldsymbol{v}_{i}^{T} \boldsymbol{x}_{0} .
$$

Equation (10) is an expression of the concept of superposition; the solution $\boldsymbol{x}(t)$ is a linear combination of the solutions associated with each eigenvector.

The qualitative stability of the nonlinear system (5) in the neighborhood of the origin is characterized by the eigenvalues of $A$. If all eigenvalues $\lambda_{i}$ have negative real parts, then the exponential term in equation $\sqrt{10}$ will decay to zero as $t \rightarrow \infty$ and the state $\boldsymbol{x}(t)$ will tend toward the origin. In such cases, the system is said to be asymptotically stable. On the other hand, if one or more eigenvalues have positive real parts, the exponential term in equation 10 will grow unbounded as $t \rightarrow \infty$, and the system is said to be unstable. Finally, if the real parts of any $\lambda_{i}$ are zero while the rest are strictly negative, a situation often referred to as marginal stability, a definitive stability characterization cannot be made based on linearization and some form of nonlinear analysis is necessary (Hirsch et al. 2003).

\section{TRANSVERSE DYNAMICS OF CONICAL SAILS ON GAUSSIAN LASER BEAMS}

The notion of stability outlined in the previous section is defined with respect to an equilibrium point. Therefore, we must first have an equilibrium point to analyze the stability of. Clearly, the full dynamics of a beamriding sail do not possess any equilibria: The sail accelerates as long as it remains on the beam. However, projecting the dynamics onto what we call the transverse subspace results in a system with an equilibrium point at the origin.

We define the transverse coordinates as those orthogonal to the beam axis. As depicted in Figure 2, the coordinates $x$ and $y$ are used to describe translation of the sail in the plane orthogonal to the beam, while the angles $\theta$ and $\phi$ are used to describe rotation of the sail about the $x$ and $y$ axes, respectively. The $x$ and $y$ components of the angular velocity vector are denoted by $\omega_{x}$ and $\omega_{y}$. The laser beam is assumed to have a radially symmetric Gaussian power distribution with full-widthat-half-maximum $W$, which can be expressed in terms of the standard deviation $\sigma$ as,

$$
W=2 \sigma \sqrt{2 \ln (2)} .
$$


In addition, we assume that the sail is axially symmetric about the $z$-axis, with mass $m$ and moments of inertia $I_{z}$ and $I_{x}=I_{y}$, that the sail is possibly spinning about the $z$-axis with angular frequency $\omega_{0}$, and that the cone angle measured relative to the $x-y$ plane is $\alpha$. To simplify the subsequent analysis, we also assume that multiple reflections of the beam do not occur. For this last assumption to hold, $\alpha$ must be less than $30^{\circ}$.

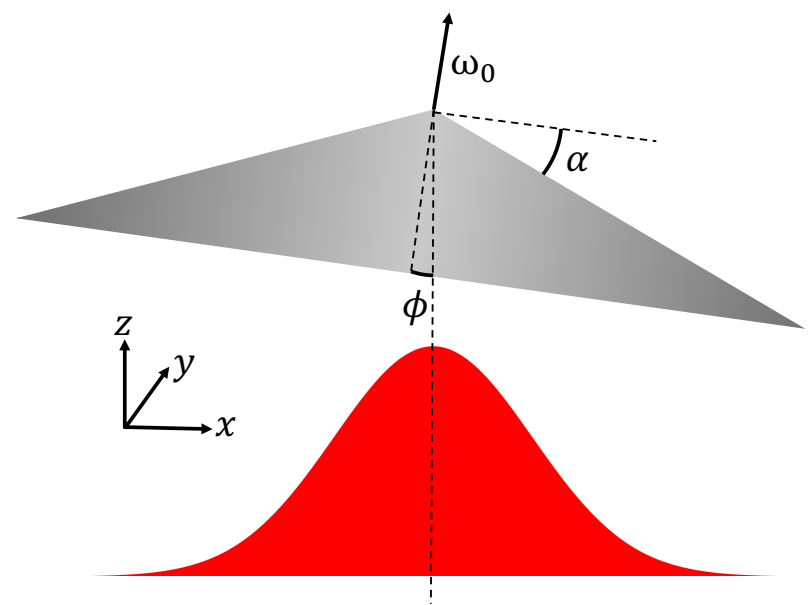

Figure 2. Schematic illustration of the geometry for a spinning conical sail riding on a Gaussian laser beam.

We can now give a formal definition of beam-riding stability as the stability of the origin with respect to the sail's transverse dynamics. In the remainder of this section we derive a linear model that approximates those dynamics for a conical sail near the center of a Gaussian laser beam.

\subsection{Translation}

From basic geometry and ray optics, the translational motion of a conical sail near the center of the beam can be approximated by the equations,

$$
\begin{gathered}
\ddot{x}=-k_{1} x+k_{2} \phi \\
\ddot{y}=-k_{1} y-k_{2} \theta
\end{gathered}
$$

where $k_{1}$ denotes the partial derivative of the sail's transverse acceleration with respect to displacements in the $x-y$ plane,

$$
k_{1}=-\frac{1}{m} \frac{\partial F_{x}}{\partial x}=-\frac{1}{m} \frac{\partial F_{y}}{\partial y},
$$

and $k_{2}$ is the partial derivative of the sail's transverse acceleration with respect to rotations about the $x$ and $y$ axes:

$$
k_{2}=\frac{1}{m} \frac{\partial F_{x}}{\partial \phi}=-\frac{1}{m} \frac{\partial F_{y}}{\partial \theta} .
$$

The first term in equation 12 describes the restoring force due to the sail's conical shape, while the second term describes the forces encountered as the sail rotates by a small angle, causing a component of the beam to be deflected in the $x-y$ plane.

An expression for $k_{1}$ in terms of the system's parameters can be derived by integrating equation (1) over a sail with a small displacement $\delta_{x}$ :

$$
\Delta F_{x}=\iint_{S} 2 \frac{P\left(x+\delta_{x}, y\right) \hat{\boldsymbol{b}} \cdot \hat{\boldsymbol{n}}(x, y)}{c} \hat{\boldsymbol{n}}_{x}(x, y) d x d y .
$$

In polar coordinates, equation (15) becomes,

$$
\begin{array}{r}
\Delta F_{x}=\int_{0}^{R} \int_{0}^{2 \pi} \frac{P_{0}}{\pi c \sigma^{2}} e^{-\left(r^{2}+2 \delta_{x} r \cos (\psi)+\delta_{x}^{2}\right) / 2 \sigma^{2}} \ldots \\
\cos (\alpha) \sin (\alpha) \cos (\psi) r d r d \psi .
\end{array}
$$

where $\psi$ is the polar angle in our coordinate system and we have used the standard deviation $\sigma$ instead of $W$ for clarity. We now retain only terms up to first order in $\delta_{x}$ :

$$
\begin{array}{r}
\Delta F_{x}=\int_{0}^{R} \int_{0}^{2 \pi} \frac{P_{0}}{\pi c \sigma^{2}} e^{-r^{2} / 2 \sigma^{2}}\left(1+\frac{\delta_{x} r}{\sigma^{2}} \cos (\psi)\right) \ldots \\
\cos (\alpha) \sin (\alpha) \cos (\psi) r d r d \psi \\
=\frac{P_{0} \delta_{x}}{2 c \sigma^{4}} \sin (2 \alpha) \int_{0}^{R} r^{2} e^{-r^{2} / 2 \sigma^{2}} d r .
\end{array}
$$

With the assumption $W \approx R$, most of the beam flux falls on the sail. Therefore we take the limit $R \rightarrow \infty$ to obtain the closed-form approximation,

$$
\Delta F_{x} \approx \frac{P_{0} \sqrt{\pi} \sin (2 \alpha)}{c \sigma 2 \sqrt{2}} \delta_{x} .
$$

Finally, the limit $\delta_{x} \rightarrow 0$ is taken and $\sigma$ is written in terms of $W$ to arrive at,

$$
k_{1}=\frac{P_{0} \sqrt{\pi \ln (2)}}{m c W} \sin (2 \alpha) .
$$

Performing similar steps, equation (2) can be integrated over the surface of a sail rotated by a small angle $\delta_{\phi}$ to find the following expression for $k_{2}$,

$$
k_{2}=\frac{P_{0}}{m c}\left(2 \cos ^{2}(\alpha)-\sin ^{2}(\alpha)-\frac{d \sqrt{\pi \ln (2)}}{W} \sin (2 \alpha)\right),
$$

where $d$ is the distance from the tip of the cone to the sail's center of mass.

\subsection{Rotation}

The angular motion of the sail near the upright orientation $\theta=\phi=0$ can be described by the kinematic equations,

$$
\begin{aligned}
& \dot{\theta}=\omega_{x}+\omega_{0} \phi \\
& \dot{\phi}=\omega_{y}+\omega_{0} \theta,
\end{aligned}
$$

which encode the fact that, as the sail tilts, a component of the $z$-axis angular velocity $\omega_{0}$ is projected onto the $x-y$ plane, along with the following dynamics:

$$
\begin{aligned}
& \dot{\omega}_{x}=-k_{3} y+k_{4} \theta-k_{5} \omega_{y} \\
& \dot{\omega}_{y}=k_{3} x+k_{4} \phi+k_{5} \omega_{x} .
\end{aligned}
$$

The constant $k_{3}$ describes the torque imparted by the beam on the sail due to small translations in the $x-y$ plane. Mathematically, it is given by,

$$
k_{3}=\frac{1}{I_{x}} \frac{\partial \tau_{x}}{\partial y}=\frac{1}{I_{y}} \frac{\partial \tau_{y}}{\partial x},
$$


where $\tau_{x}$ and $\tau_{y}$ are the $x$ and $y$ components of the torque vector. Proceeding in the same fashion as with $k_{1}$ and $k_{2}$, equation (2) is integrated over the surface of a sail with a small displacement. With the approximation $R \rightarrow \infty$, we arrive at,

$$
k_{3}=\frac{P_{0}}{c I_{x}}\left(2-\frac{d \sqrt{\pi \ln (2)}}{W} \sin (2 \alpha)\right) .
$$

The first term in equation 24 describes what would happen if the beam were a single infinitesimally thin ray and the sail were flat, while the second term accounts for the finite width of the beam and the angle of the cone.

The constant $k_{4}$ describes a torque due to one side of the sail encountering greater photon pressure than the other as the sail rotates:

$$
k_{4}=\frac{1}{I_{x}} \frac{\partial \tau_{x}}{\partial \theta}=\frac{1}{I_{y}} \frac{\partial \tau_{y}}{\partial \phi} .
$$

As before, we integrate equation (2); this time over the surface of a sail that has been rotated by a small angle. Once again, we take the limit $R \rightarrow \infty$ to find the following closed-form result:

$$
\begin{array}{r}
k_{4}=\frac{P_{0}}{c I_{x}}\left(d\left(2 \cos (\alpha)-3 \sin ^{2}(\alpha)\right)-d^{2} \frac{\sqrt{\pi \ln (2)}}{W} \sin (2 \alpha)\right. \\
\left.-\frac{W \sqrt{\pi}}{4 \sqrt{\ln (2)}}\left(\sin (\alpha)-\sin ^{2}(\alpha) \tan (\alpha)\right)\right) .
\end{array}
$$

Lastly, the constant $k_{5}$ appearing in equation 22 captures gyroscopic effects due to the sail's spin about the $z$-axis. It can be derived from Euler's equation (4) by making the assumption that $\omega_{0}$ is much greater than both $\omega_{x}$ and $\omega_{y}$ (Hughes 2004). In terms of the system's parameters, its value is

$$
k_{5}=\omega_{0}\left(\frac{I_{x}-I_{z}}{I_{x}}\right) .
$$

Assembling equations 12 and 22 into the generic form of equation (7) gives the following linear system:

$$
\left[\begin{array}{c}
\dot{x} \\
\dot{y} \\
\dot{\theta} \\
\dot{\phi} \\
\ddot{x} \\
\ddot{y} \\
\dot{\omega}_{x} \\
\dot{\omega}_{y}
\end{array}\right]=\left[\begin{array}{cccccccc}
0 & 0 & 0 & 0 & 1 & 0 & 0 & 0 \\
0 & 0 & 0 & 0 & 0 & 1 & 0 & 0 \\
0 & 0 & 0 & \omega_{0} & 0 & 0 & 1 & 0 \\
0 & 0 & -\omega_{0} & 0 & 0 & 0 & 0 & 1 \\
-k_{1} & 0 & 0 & k_{2} & 0 & 0 & 0 & 0 \\
0 & -k_{1} & -k_{2} & 0 & 0 & 0 & 0 & 0 \\
0 & -k_{3} & k_{4} & 0 & 0 & 0 & 0 & k_{5} \\
k_{3} & 0 & 0 & k_{4} & 0 & 0 & -k_{5} & 0
\end{array}\right]\left[\begin{array}{c}
x \\
y \\
\theta \\
\phi \\
\dot{x} \\
\dot{y} \\
\omega_{x} \\
\omega_{y}
\end{array}\right]
$$

\section{STABILITY OF CONICAL SAILS}

Forming the full eigendecomposition of the matrix in (28), which we will refer to as $A_{\text {cone, }}$ is quite unwieldy analytically. Instead, we infer information about its eigenvalues based on its structure. First, we note that $A_{\text {cone }}$ is traceless. Since the trace of a matrix is equal to the sum of its eigenvalues, it is not possible for all of the eigenvalues of $A_{\text {cone }}$ to have negative real parts. As a result, asymptotic stability cannot be achieved, and the best that can be hoped for is to arrange all of the eigenvalues to lie on the imaginary axis. While we pursue such marginal stability results in this section, it is important to keep in mind that they do not give sufficient conditions for stability of the full nonlinear system, and must be verified by numerical simulation.

\subsection{The Non-Spinning Case}

In the non-spinning case in which $\omega_{0}=k_{5}=0$, equation (28) takes the form of an undamped oscillator,

$$
\ddot{\boldsymbol{x}}+K \boldsymbol{x}=0,
$$

where $\boldsymbol{x}=\left[\begin{array}{llll}x & y & \theta & \phi\end{array}\right]^{T}$ and $-K$ is the lower left $4 \times 4$ block of $A_{\text {cone }}$ :

$$
K=\left[\begin{array}{cccc}
k_{1} & 0 & 0 & -k_{2} \\
0 & k_{1} & k_{2} & 0 \\
0 & k_{3} & -k_{4} & 0 \\
-k_{3} & 0 & 0 & -k_{4}
\end{array}\right] .
$$

Thanks to the symmetry of the beam-riding system, $K$ can be brought into block-diagonal form by a simple permutation of the coordinates such that $\boldsymbol{x}^{\prime}=\left[\begin{array}{lll}x & \theta & y\end{array}\right]^{T}$ :

$$
K^{\prime}=\left[\begin{array}{cccc}
k_{1} & -k_{2} & 0 & 0 \\
-k_{3} & -k_{4} & 0 & 0 \\
0 & 0 & k_{1} & k_{2} \\
0 & 0 & k_{3} & -k_{4}
\end{array}\right]
$$

The eigenvalues of $K$ can now be found in closed form by analyzing each $2 \times 2$ block of $K^{\prime}$ separately, resulting in:

$$
\begin{aligned}
\lambda_{1}= & \lambda_{2}=\frac{1}{2}\left(k_{1}-k_{4}\right. \\
& \left.+\sqrt{\left(k_{1}-k_{4}\right)^{2}+4 k_{1} k_{4}+4 k_{2} k_{3}}\right) \\
\lambda_{3}= & \lambda_{4}=\frac{1}{2}\left(k_{1}-k_{4}\right. \\
& \left.-\sqrt{\left(k_{1}-k_{4}\right)^{2}+4 k_{1} k_{4}+4 k_{2} k_{3}}\right) .
\end{aligned}
$$

In analogy with the scalar case, the "spring constant" or "stiffness" matrix $K$ must have positive real eigenvalues for the system to be marginally stable. From equation (33), a necessary condition is thus,

$$
k_{1} k_{4}+k_{2} k_{3}<0 \text {. }
$$

This condition is plotted in Figure 3 as a function of the cone angle, $\alpha$, and the distance between the cone tip and the center of mass, $d$, normalized by the cone radius $R$. A line showing the cone height, which serves as an upper bound on, $d$ is also plotted.

Figure 3 indicates that a simple conical sail cannot achieve marginal stability. Instead, the center of mass of the sail must lie beneath the cone by a distance equal to several times its radius. While one can imagine mechanical solutions that could place the center of mass beneath the base of the cone, such as the rigid pendulum suggested by (Chahine et al. 2003), they introduce serious practical ditficulties. First, any such structure will necessarily be exposed to the laser beam - a very challenging thermal environment. Second, the flexible modes of the structure would have to be carefully considered. A stiff structure, in which the natural frequencies associated with flexible modes are much higher than those 


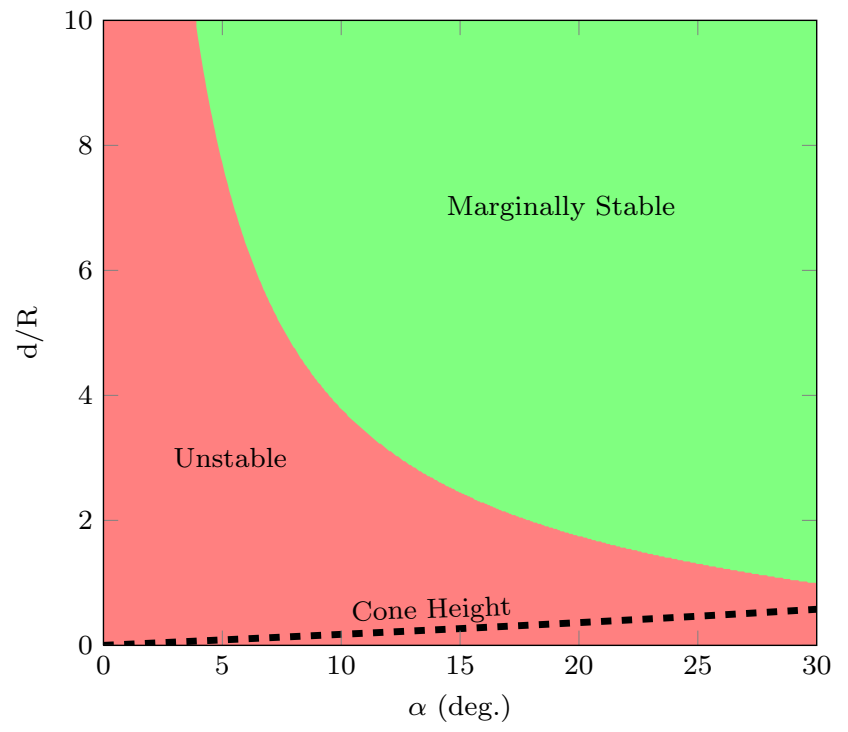

Figure 3. Stability regions in the space of sail parameters $\alpha$ (cone angle) and $d / R$ (center of mass location normalized by the cone radius). The dashed line marks the cone height.

associated with rigid body modes, is desirable from a stability standpoint. However, from an engineering standpoint, such a structure is likely to be heavy and may add significant mass to the spacecraft, making it difficult to achieve the desired final velocity with a practical laser system.

\subsection{The Spinning Case}

We now turn to the spinning case in which $\omega_{0}$ and $k_{5}$ are both non-zero. First, we note that it is possible to achieve marginal stability, that is, placement of all eigenvalues of $A_{\text {cone }}$ on the imaginary axis, by an appropriate choice of $\omega_{0}$. However, the situation is somewhat more subtle than might be expected from an analysis of the transverse dynamics alone.

While equation (28) captures gyroscopic precession and nutation effects, it implicitly assumes that the sail's angular momentum vector $\boldsymbol{\ell}=I \boldsymbol{\omega}$ is perfectly aligned with the beam axis $\hat{\boldsymbol{b}}$. If the sail has an initial $\boldsymbol{\ell}$ that is not in perfect alignment with the beam axis, the $\mathrm{SO}(2)$ symmetry of the system is broken and the equilibrium point in the transverse dynamics disappears. A stability analysis in the sense of Section 2.2 is therefore misleading.

A qualitative physical understanding of the situation can be gained by recalling the behavior of a rigid body undergoing precession. Viewed in an inertial reference frame, the body's angular velocity vector $\boldsymbol{\omega}$ traces out a cone centered on its angular momentum vector (Figure 4). In the case of a conical sail with $\ell$ parallel to the beam axis, one can see that it should be possible, with a sufficiently high spin frequency $\omega_{0}$, for perturbing forces to "average out" over a precession period, resulting in a marginally stable system. However, if $\ell$ is not exactly parallel to the beam axis, the average force on the sail over a precession period will have a component in the $x-y$ plane, pushing the sail off the beam.

In mathematical terms, the equilibrium point in the sail's transverse dynamics is not structurally stable. It exists only for very particular choices of $\ell$, the set of

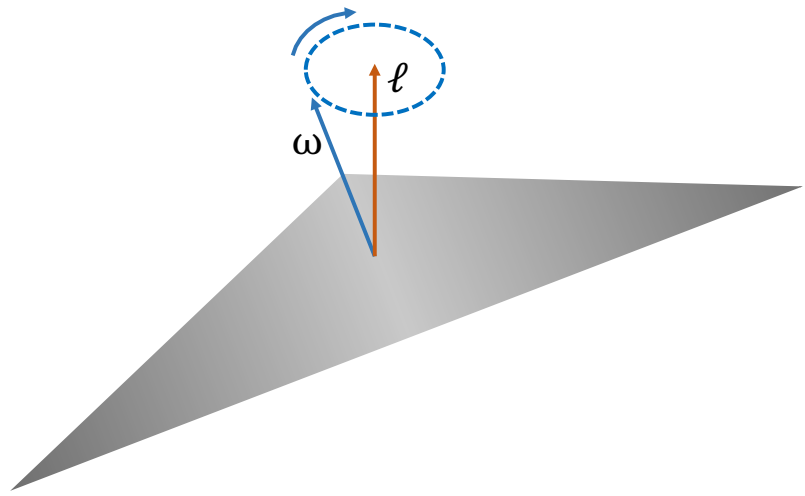

Figure 4. Illustration of the effect of gyroscopic precession on the angular velocity vector, $\omega$, of the sail for an angular momentum $\ell$.

which has zero Lebesgue measure. In practical terms, it is not possible to achieve perfect alignment of the sail's angular momentum vector with the beam axis. Therefore, we cannot expect a spinning conical sail to achieve stable beam riding.

\section{SPHERICAL SHELL DESIGN FOR THE SAIL}

We now propose an alternative beam-riding architecture with more favorable stability properties. Upon close inspection of the matrix in equation (28), it is clear that the instabilities found in the previous section are rooted in a coupling between the translation and rotation degrees of freedom of the sail. Motivated by this observation, we analyze a spherical shell configuration for the sail, whose symmetry eliminates such coupling.

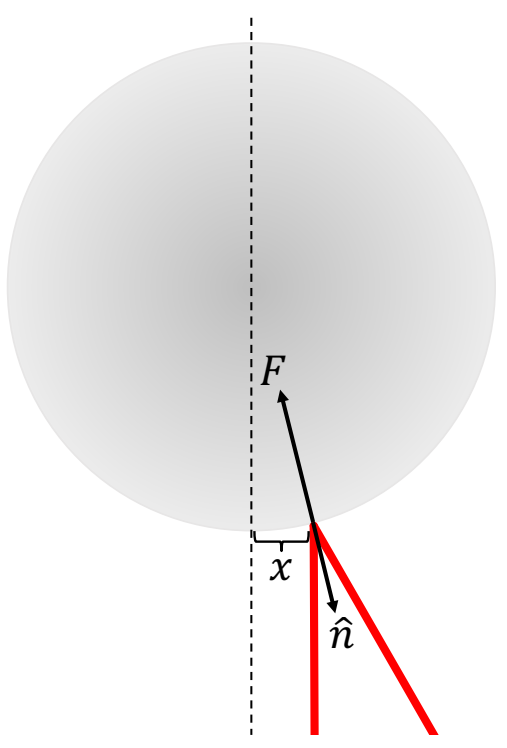

Figure 5. Schematic illustration of the geometry for a reflective sail in the shape of a thin spherical shell. The force $\boldsymbol{F}$ is always along the surface normal direction $\hat{\boldsymbol{n}}$.

Figure 5 depicts the force imparted on a reflective spherical sail by a light ray offset from the center of the sphere. The ray clearly does not produce a restoring force, instead pushing the sphere farther away from the beam axis. Any unimodal beam profile, like the Gaussian studied in the previous section, will have a similar effect, resulting in an unstable system. 
If the beam is instead allowed to be multimodal, stable beam riding becomes possible. Figure 6 depicts a beam profile composed of a sum of four Gaussians. An appropriately sized spherical sail perturbed from the center of such a composite beam will experience a restoring force pushing it back toward the center due to the increased power in the sides of the beam. Note that there are no torques applied to the sphere since all forces are directed toward the sphere's center.

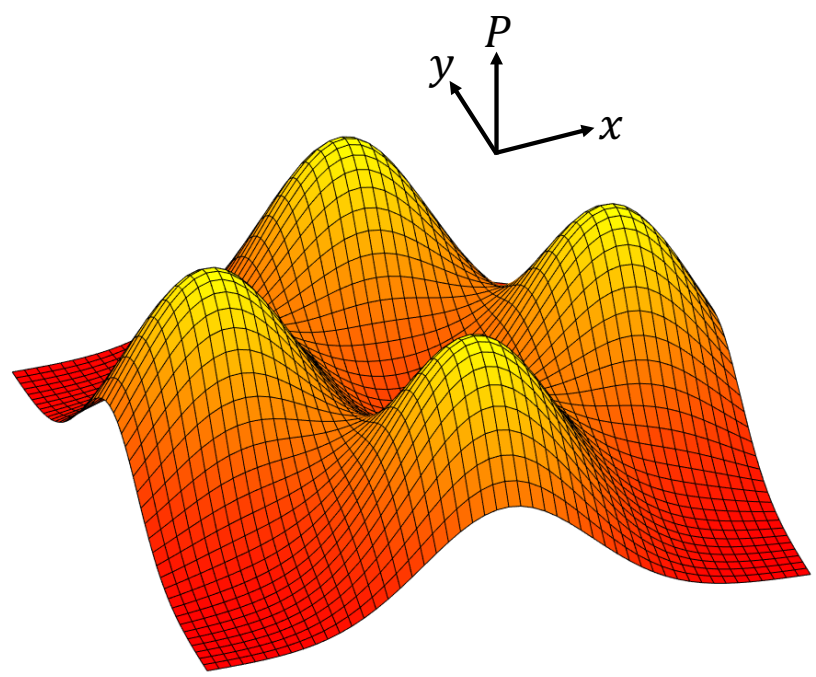

Figure 6. Multimodal beam profile composed of four Gaussian laser beams.

The linearized transverse dynamics for this beam riding configuration take the form,

$$
\left[\begin{array}{c}
\dot{x} \\
\dot{y} \\
\dot{\theta} \\
\dot{\phi} \\
\ddot{x} \\
\ddot{y} \\
\ddot{\theta} \\
\ddot{\phi}
\end{array}\right]=\left[\begin{array}{cccccccc}
0 & 0 & 0 & 0 & 1 & 0 & 0 & 0 \\
0 & 0 & 0 & 0 & 0 & 1 & 0 & 0 \\
0 & 0 & 0 & 0 & 0 & 0 & 1 & 0 \\
0 & 0 & 0 & 0 & 0 & 0 & 0 & 1 \\
-k_{1} & 0 & 0 & 0 & 0 & 0 & 0 & 0 \\
0 & -k_{1} & 0 & 0 & 0 & 0 & 0 & 0 \\
0 & 0 & 0 & 0 & 0 & 0 & 0 & 0 \\
0 & 0 & 0 & 0 & 0 & 0 & 0 & 0
\end{array}\right]\left[\begin{array}{c}
x \\
y \\
\theta \\
\phi \\
\dot{x} \\
\dot{y} \\
\dot{\theta} \\
\dot{\phi}
\end{array}\right],
$$

where, once again,

$$
k_{1}=-\frac{1}{m} \frac{\partial F_{x}}{\partial x}=-\frac{1}{m} \frac{\partial F_{y}}{\partial y} .
$$

As expected, there is no coupling between translation and rotation, allowing 35 to be separated into two independent linear systems:

$$
\left[\begin{array}{c}
\dot{x} \\
\dot{y} \\
\ddot{x} \\
\ddot{y}
\end{array}\right]=\left[\begin{array}{cccc}
0 & 0 & 1 & 0 \\
0 & 0 & 0 & 1 \\
-k_{1} & 0 & 0 & 0 \\
0 & -k_{1} & 0 & 0
\end{array}\right]\left[\begin{array}{l}
x \\
y \\
\dot{x} \\
\dot{y}
\end{array}\right],
$$

and

$$
\left[\begin{array}{c}
\dot{\theta} \\
\dot{\phi} \\
\ddot{\theta} \\
\ddot{\phi}
\end{array}\right]=\left[\begin{array}{llll}
0 & 0 & 1 & 0 \\
0 & 0 & 0 & 1 \\
0 & 0 & 0 & 0 \\
0 & 0 & 0 & 0
\end{array}\right]\left[\begin{array}{c}
\theta \\
\phi \\
\dot{\theta} \\
\dot{\phi}
\end{array}\right] .
$$

Since we are concerned with keeping the sail centered on the beam, we will focus on the translation dynamics.
The eigenvalues of the matrix in equation (37) can be readily found in closed form:

$$
\begin{gathered}
\lambda_{1}=\lambda_{2}=i \sqrt{k_{1}} \\
\lambda_{3}=\lambda_{4}=-i \sqrt{k_{1}} .
\end{gathered}
$$

All eigenvalues lie on the imaginary axis, therefore the linearized system is marginally stable, and a definitive stability characterization cannot be made for the full nonlinear system.

To obtain more conclusive stability information, the beam profile of Figure 6 is discretized on a grid and the forces on the sail are evaluated at each grid point. Since the transverse forces $\boldsymbol{F}_{\perp}$ depend only on the sail's position in the $x-y$ plane and are not functions of velocity or time, they are conservative (Goldstein et al. 2001). As a result, they can be associated with a scalar potential function $V(x, y)$ such that $\boldsymbol{F}_{\perp}=-\nabla V(x, y)$. We then compute this potential function numerically, as depicted in Figure 7

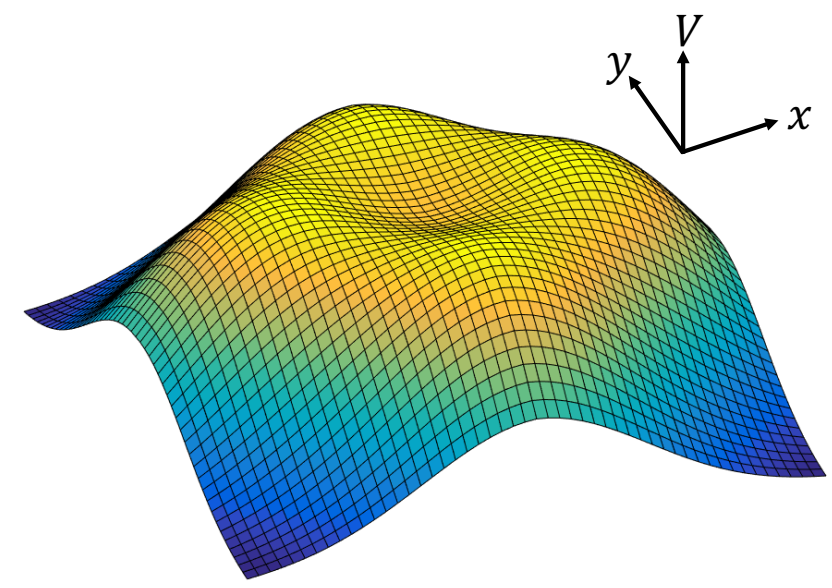

Figure 7. Potential function $V(x, y)$ for the transverse dynamics of a spherical sail riding on the beam profile shown in Figure6

Based on Figure 7, it is clear that there is a basin of attraction surrounding the center of the beam. As long as the sail's initial conditions lie within this basin and its total energy is below the energy associated with the rim of the basin (which can be calculated numerically for any parameters of interest), the sail will remain trapped in the basin. Physically, the sail will oscillate around the center of the beam, but the amplitude of the oscillations will remain bounded and the sail will remain on the beam. While this does not meet the definition of asymptotic stability presented in Section 2, it does meet the looser requirements of Lyapunov stability (Khalil 2002).

\section{NUMERICAL SIMULATIONS}

We demonstrated the stability of the spherical sail riding on the composite beam profile shown in Figure 6 through two numerical simulations. The integrals in equations (1) and (2) were approximated by discretizing the beam shown in Figure 6 into a grid of $50 \times 50$ rays. The path of each ray was then traced as it intersected the sail and reflected off of its surface. The net change in momentum of each ray was calculated, and the resulting forces and torques were applied to the sail. The differential equations (3) and (4) were then integrated 
forward in time using the standard fourth-order RungeKutta method with a time step of 0.001 seconds. The parameters used in our simulations follow the Starshot design, with a beam power $P=100 \mathrm{GW}$, a sail mass $m=10 \mathrm{~g}$, a sphere radius $R=1 \mathrm{~m}$, a width of each constituent Gaussian in the beam of $W=1 \mathrm{~m}$, and a distance of $1 \mathrm{~m}$ between the center of each constituent Gaussian and the overall beam center.

Figure 8 shows the components of the sail's position vector during a short simulation with an initial offset of $5 \mathrm{~cm}$ in both the $x$ and $y$ components of the position vector and zero initial velocity. The sail's position in the $x-y$ plane oscillates with a frequency of roughly $11 \mathrm{~Hz}$ but, as predicted, remains bounded.
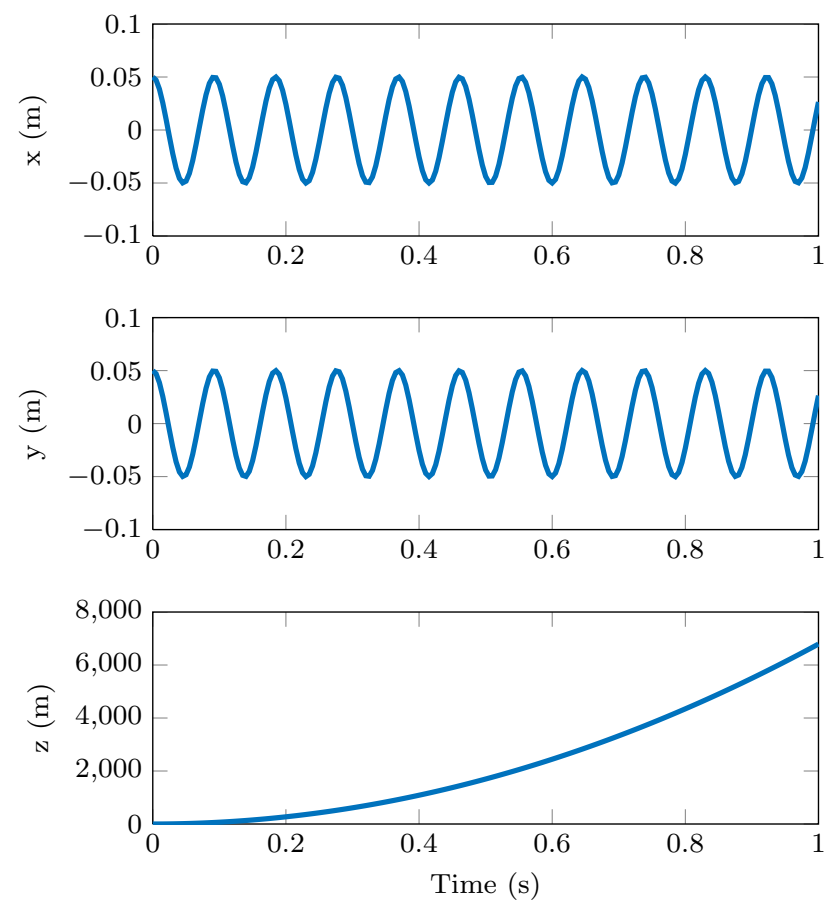

Figure 8. Sail position during a beam-riding simulation.

Figure 9 shows the components of the sail's position vector during a longer simulation in which white noise is added to the rays making up the laser beam to simulate perturbations due to, for example, atmospheric effects. The average power of the noise applied to each ray is chosen to be $10 \%$ of that ray's nominal power. While perturbations of the beam clearly excite transverse oscillatory motion of the sail, the sail remains in the stable basin of attraction over a time scale of minutes, which is the time scale needed for it to achieve a sizeable fraction of the speed of light along the $z$-axis.

In general, noise will add energy to the transverse modes of the system. The total energy of the system will execute a random walk and, after sufficient time, will exceed the energy associated with the rim of the potential well, causing the sail to leave the beam. This "exit time," which depends on the beam power and shape, as well as the power spectral density of the noise, will be an important consideration in the design of a realistic laser-sail system.
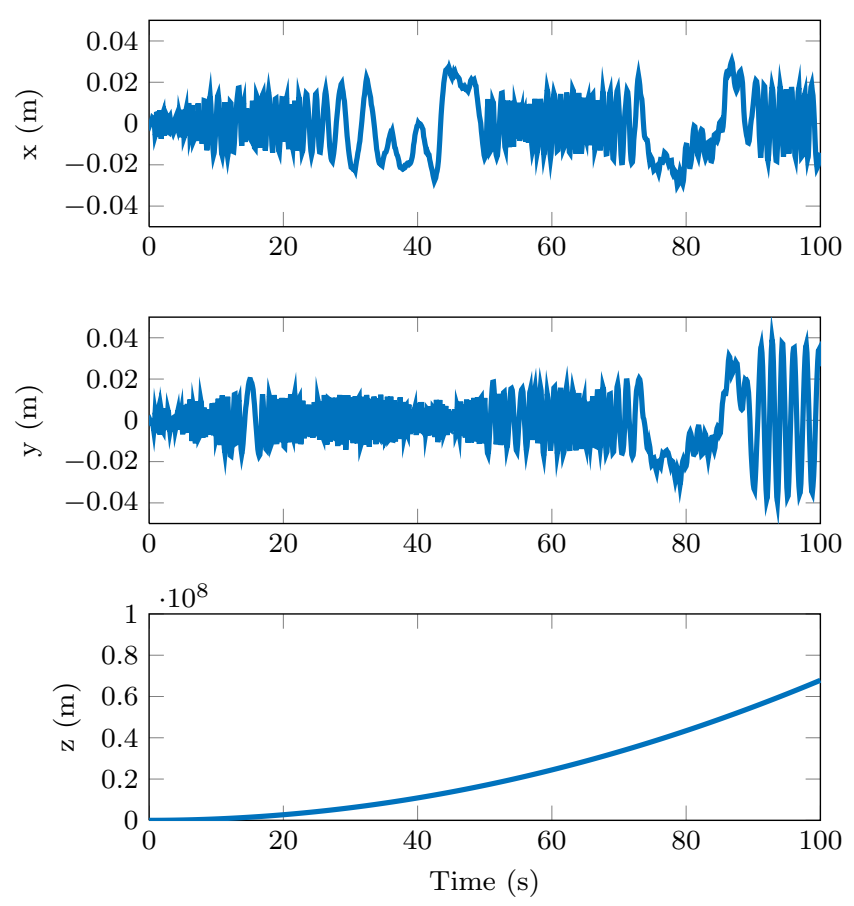

Figure 9. Sail position during a simulation with white noise of average power equal to $10 \%$ of the nominal beam power.

\section{DISCUSSION}

We have presented a passively stable laser-sail architecture that is capable of beam riding without active feedback control. The proposed design makes use of a spherical shell sail and a multimodal beam profile. While we have focused on a particular beam profile composed of a sum of four Gaussians, many others are possible, including sums of three or more Gaussians and radially symmetric ring-like profiles.

There are several effects which were not accounted for in this study, but which are likely to be important in the practical implementation of a laser-sail system. Perhaps most importantly, we have assumed a perfectly rigid sail. In practice, the sail will have flexible structural modes which may impact its beam-riding dynamics. Deformation of the sphere's surface could cause non-zero torques on the sail, but could also result in favorable energy damping, which would reduce the amplitude of oscillations about the center of the beam. It may also be possible to pressurize the interior of the sphere with a gas or actively control the structure with actuators to alter both its stiffness and damping properties.

\section{ACKNOWLEDGEMENTS}

The authors acknowledge support from the Breakthrough Prize Foundation, and are grateful to the Starshot group at Harvard for comments on the content of this paper.

\section{REFERENCES}

F. Zander, Problems of flight by jet propulsion: Interplanetary flights, NASA Technical Translation F-147 (1964).

R. L. Forward, Journal of Spacecraft and Rockets 21, 187 (1984) G. Marx, Nature 211, 22 (1966)

J. Redding, Nature 213, 588 (1967).

W. E. Moeckel, Journal of Spacecraft and Rockets 9, 942 (1972). 
R. F. Weiss, A. N. Pirri, and N. H. Kemp, Astronautics and Aeronautics 17, 50 (1979).

P. Lubin, Journal of the British Interplanetary Society (to be published).

B. Wie, Journal of Guidance, Control, and Dynamics 27, 526 (2004a).

B. Wie, Journal of Guidance, Control, and Dynamics 27, 536 (2004b).

S. W. Smith, H. Song, J. R. Baker, J. Black, and D. M. Muheim, in Proceedings of the 46th AIAA/ASME/ASCE/AHS/ASC Structures, Structural Dynamics 85 Materials Conference, Austin, Texas, American Institute of Aeronautics and Astronautics (2005).

Y. Mimasu, T. Yamaguchi, M. Matsumoto, M. Nakamiya, R. Funase, and J. Kawaguchi, Advances in Space Research 48, 1810 (2011).

M. Polites, J. Kalmanson, and D. Mangus, Proceedings of the Institution of Mechanical Engineers, Part G: Journal of Aerospace Engineering 222, 53 (2008).

E. Chahine, C. Abdallah, D. Georgiev, and E. Schamiloglu, in AIAA Space 2003 Conference and Exposition (2003).
E. Schamiloglu, C. T. Abdallah, K. A. Miller, D. Georgiev, J. Benford, G. Benford, and G. Singh, AIP Conference Proceedings 552 (2001).

J. Benford, G. Benford, O. Gornostaeva, E. Garate, M. Anderson, A. Prichard, and H. Harris, AIP Conference Proceedings 608 (2002).

G. Benford, O. Gornostaeva, and J. Benford, AIP Conference Proceedings 664 (2003).

T. Kailath, Linear Systems, Information and System Sciences Series (Prentice-Hall, 1980).

M. Hirsch, S. Smale,, and R. Devaney, Differential Equations, Dynamical Systems, and an Introduction to Chaos, Pure and Applied Mathematics (Elsevier, 2003).

P. Hughes, Spacecraft Attitude Dynamics, Dover Books on Aeronautical Engineering (Dover Publications, Mineola, New York, 2004).

H. Goldstein, C. Poole, and J. Safko, Classical Mechanics, 3rd ed. (Addison Wesley, San Francisco, 2001).

H. Khalil, Nonlinear Systems, Pearson Education (Prentice Hall, 2002) 